\title{
Effect of Entropy Guided Low Flow Desflurane Anaesthesia on Laryngeal Mask Airway Removal Time in Children Undergoing Elective Ophthalmic Surgery - A Prospective, Randomized, Comparative Study
}

Mishra $\mathbf{S}^{1}$, Sinha $\mathrm{R}^{1}$, Ray $\mathrm{BR}^{1}$, Darlong $\mathrm{V}^{1}$, Pandey $\mathrm{RK}^{1}$, Punj $\mathrm{J}^{1}$

Department of Anaesthesiology, Pain Medicine and Critical Care, All India Institute of Medical Sciences, New Delhi

\section{INTRODUCTION}

- In children, entropy \& Bi spectral index (BIS) guided titration of inhalational anaesthetic agent like isoflurane \& sevoflurane leads to faster recovery after anaesthesia.

- However, role of entropy \& low flow anaesthesia in recovery following desflurane anaesthesia is not known in children.

- We assessed effect of entropy on Laryngeal Mask Airway (LMA) removal in children undergoing ophthalmic surgery using low flow desflurane anaesthesia.

- We also evaluated effect of entropy on consumption of desflurane in children.

\section{METHODOLOGY}

- After ethics committee approval, parental consent \& assent, 80 ASA grade I-II children, aged 2-14 years, undergoing elective ophthalmic surgery were randomized into entropy guided (group E) and minimum alveolar concentration (MAC) guided group (group M).

- After induction of general anaesthesia \& muscle relaxation, LMA was inserted and anaesthesia was maintained using oxygen, air(FiO2 0.5) \& desflurane using low fresh gas flow $(0.8 \mathrm{~L} / \mathrm{min})$.

- In Entropy group, desflurane was titrated to maintain state entropy (SE) value of 40-60.

- In MAC group, desflurane was titrated to maintain a MAC value between 1-1.3.

- Intraoperatively, Paracetamol $15 \mathrm{mg} / \mathrm{kg}$ was administered for analgesia and Ondansetron $100 \mu \mathrm{g} / \mathrm{kg}$ for PONV prophylaxis.

- We compared LMA removal time (time from switching off desflurane at the end of surgery till removal of LMA), hemodynamic parameters, uptake \& consumption of desflurane between both the groups.

- Hemodynamic parameters, ventilator parameters, entropy and MAC values were compared between both the groups intraoperatively \& postoperatively.

- We also compared PONV, emergence delirium, pain in postoperative period and interventions used for them i.e. metoclopramide, midazolam and fentanyl respectively.

\section{RESULTS}

- The groups were comparable with reference to age, weight, sex, indication of surgery \& duration of procedure.

- There was significant decrease in LMA removal time in group $E$ (4.34 \pm 2.03 minutes) compared to group $M(8.8 \pm 2.33$ minutes $)$ $(\mathrm{p}<0.0001)$.

- Consumption of desflurane was also significantly less in group $E$ $(25.3 \pm 8.11 \mathrm{ml})$ compared to group $M(18.7 \pm 5.07)(p<0.0001)$.

- Intraoperatively, entropy values were significantly lower in group $M$, whereas MAC values and end tidal desflurane were found to be significantly lower in group $\mathrm{E}$.

- No difference was seen in PONV, emergence delirium, postoperative pain and other interventions in both the groups.
Response Entropy (RE)

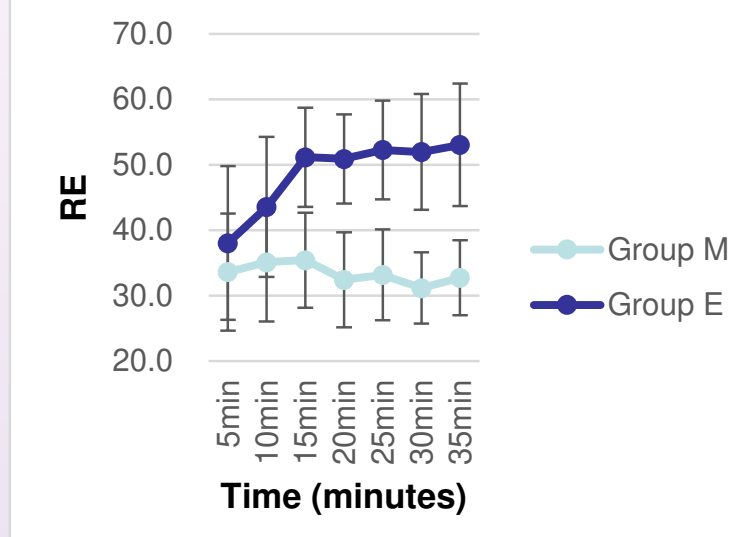

State Entropy (SE)
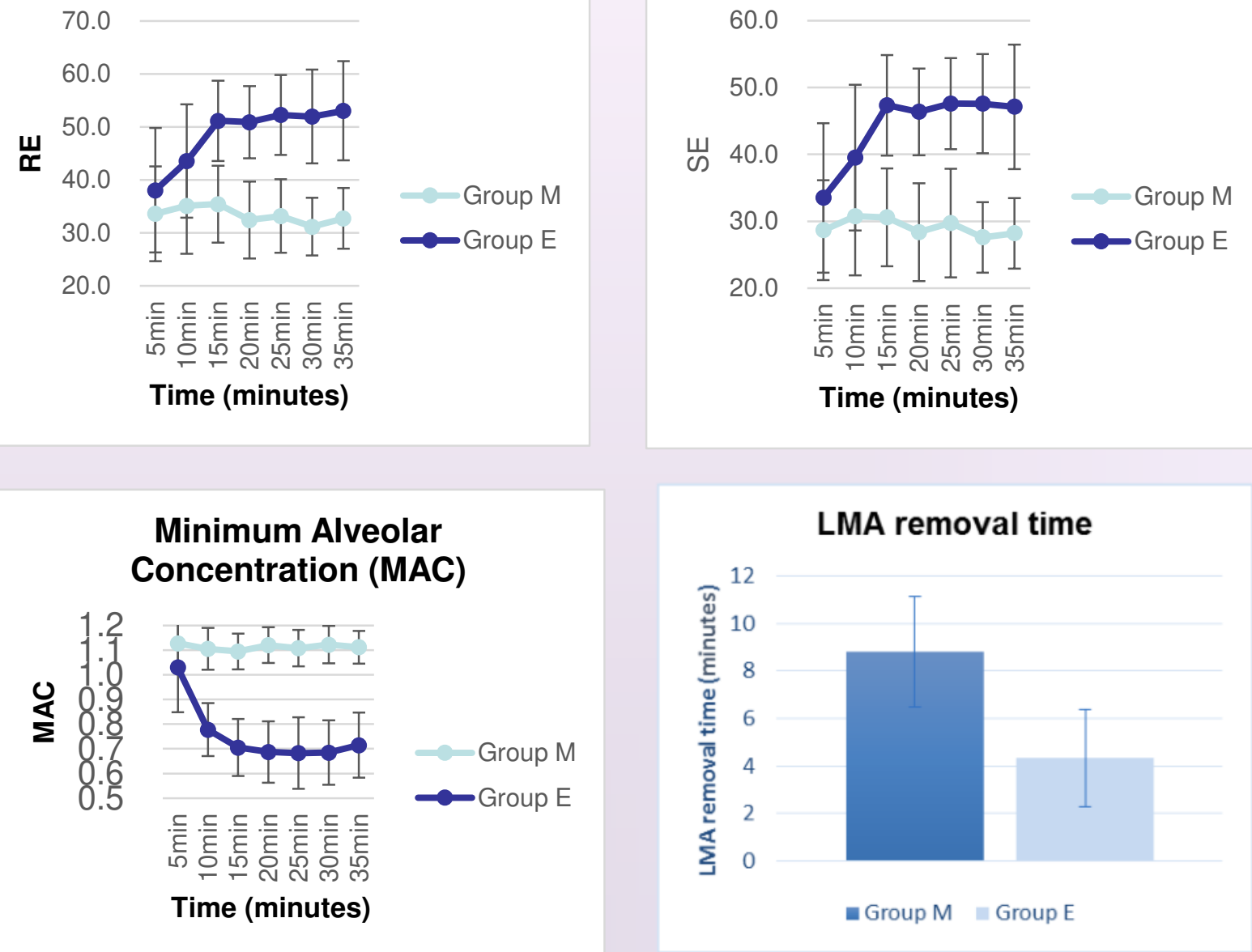

LMA removal time

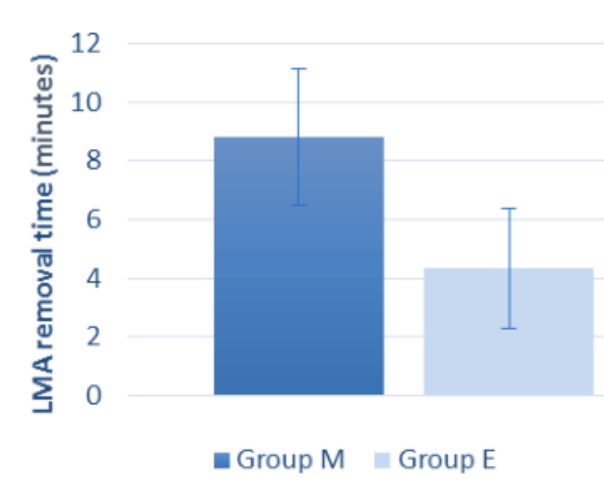

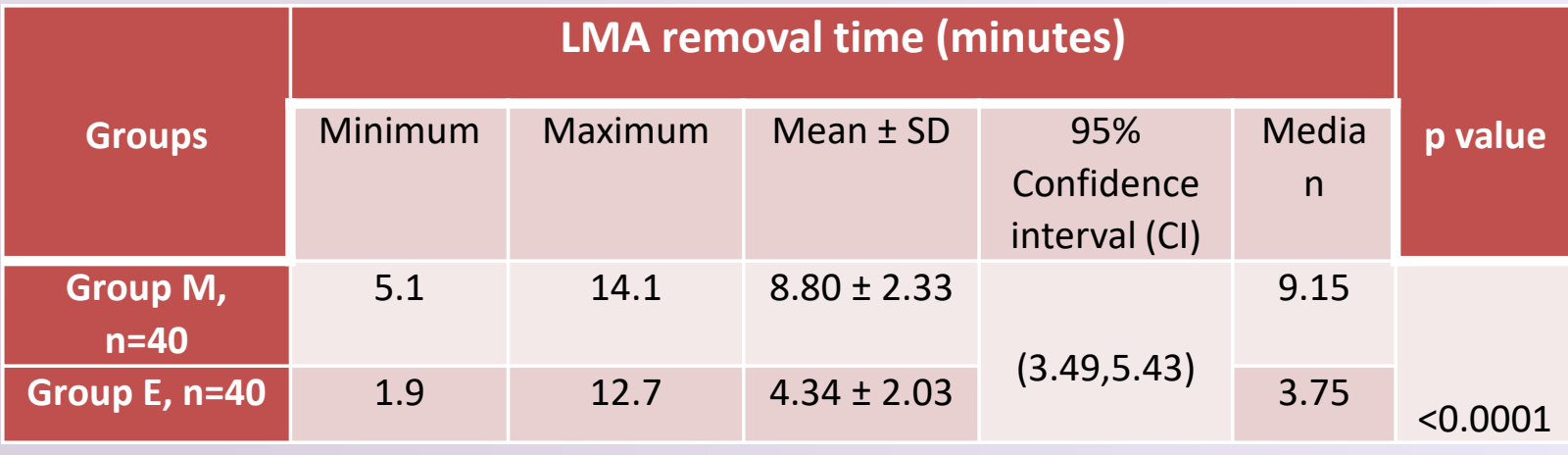

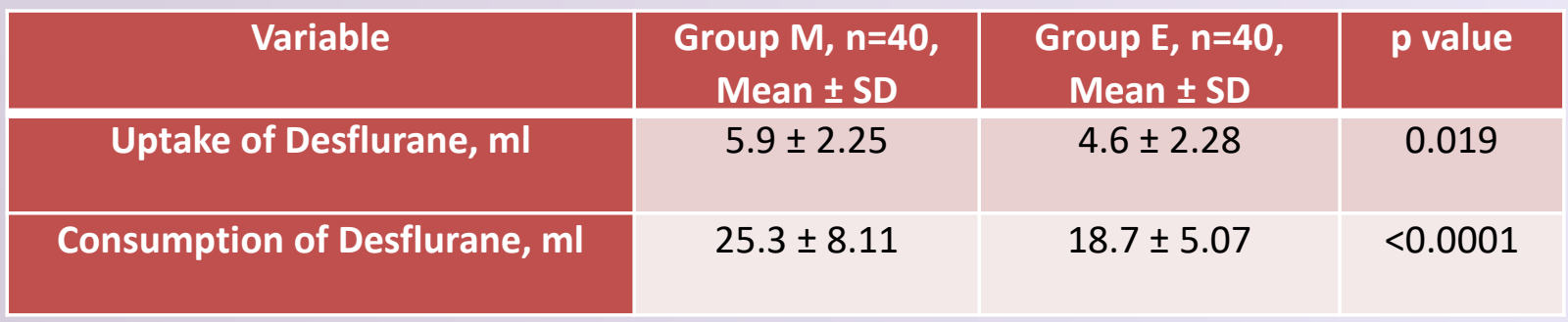

\section{DISCUSSION}

- We obtained a $50 \%$ reduction in LMA removal time with a difference of 4.46 minutes when desflurane was titrated using entropy values compared to MAC values.

- Talawar $P$ et al \& Choi et al ${ }^{(1,2)}$ also found reduction in awakening time with the entropy use for isoflurane (2min) \& sevoflurane $(3 \mathrm{~min})$ titration respectively. However they did not consider the reduction in time to be clinically significant.

- In a high volume centre, a difference of 4.46 minutes when extrapolated to the number of cases done in a day in OT will result in clinically relevant difference.

- The reason of this difference may be the use of desflurane, low flow anaesthesia and no use of premedication.

- Wu et $\mathrm{al}^{(3)}$ found a reduction in consumption of sevoflurane when titrated using entropy.

\section{CONCLUSION}

- Entropy guided low flow anaesthesia is associated with faster awakening and reduced consumption of desflurane in children 2-14 years of age. 